\title{
Driving and Dissipation of Solar-Wind Turbulence: What is the Evidence?
}

\author{
Charles W. Smith * and Bernard J. Vasquez \\ Physics Department and Space Science Center, Morse Hall, University of New Hampshire, Durham, NH, United States
}

Fifty years of solar wind observations have provided extensive data that drives an evolving view of the fundamental nature and dynamics of the magnetic, velocity, and density fluctuations that are ubiquitous throughout the heliosphere. Despite the ongoing examination of ever improving data, fundamental questions remain unanswered because there are very few multi-point measurements from a sufficient number of spacecraft in close proximity to fully resolve the three-dimensional dynamics that are at the heart of the problem. Simulations provide new insights and new questions, but most simulations sacrifice one aspect of plasma physics in order to address another. Computers and computational methods remain insufficient to simulate fully compressive, fully nonlinear, collisionless plasma dynamics with sufficient spatial range and dimension to be considered a complete description of solar wind turbulence. For these reasons, there remain multiple divergent opinions as to the underlying dynamics of solar wind turbulence,

\section{OPEN ACCESS}

Edited by:

Joseph Eric Borovsky, Space Science Institute, United States

Reviewed by: Chuanyi Tu, Peking University, China Francesco Malara, University of Calabria, Italy

*Correspondence: Charles W. Smith Charles.Smith@unh.edu

Specialty section: This article was submitted to Space Physics, a section of the journal Frontiers in Astronomy and Space Sciences

Received: 29 September 2020 Accepted: 11 December 2020 Published: 25 January 2021

Citation:

Smith CW and Vasquez BJ (2021) Driving and Dissipation of Solar-Wind Turbulence: What is the Evidence? Front. Astron. Space Sci. 7:611909. doi: 10.3389/fspas.2020.611909 dissipation, and the observed heating of the thermal plasma. We review observations of solar wind turbulence in so far as they contribute to an understanding of solar wind heating through the existence of energy reservoirs, the dynamics that move energy from the reservoirs to the dissipation scales, and the conversion into heat of energy associated with coherent fluctuations.

Keywords: instabilities, magnetic fields, solar wind, sun: heliosphere, turbulence, waves

\section{INTRODUCTION}

For decades the fundamental nature of fluctuations in the solar wind has been the object of debate. Two schools of thought have structured that debate. In the first, fluctuations are described as waves, most often solutions to the linearized dynamical equation, and the leading wave form has been Alfvén waves (Coleman, 1966; Völk and Alpers, 1973; Denskat and Burlaga, 1977; Heinemann and Olbert, 1980). In the second, fluctuations are described as being the result of diverse nonlinear dynamics that are the magnetized plasma equivalent of traditional hydrodynamic turbulence (Coleman, 1968; Bavassano et al., 1982; Matthaeus and Goldstein, 1982; Goldstein et al., 1995).

Early formulations of these ideas were based primarily on the observed power spectrum, fluctuation anisotropy, and cross-field correlation (Belcher and Davis, 1971). The view evolved and became dominant that the fluctuations originated with noncompressive waves in the subAlfvénic region of the solar atmosphere. These waves then propagate outward and across the point where the flow becomes super-Alfvénic. This transition acts as a filter for waves propagating at the Alfvén speed resulting solar wind oscillations that are dominated by outward-propagating Alfvén waves. This explains the fact that fluctuations (both magnetic and kinetic) tend to be noncompressive and transverse to the local mean magnetic field with a reproducible power spectrum that reflects the turbulence in the collision-dominated corona. 


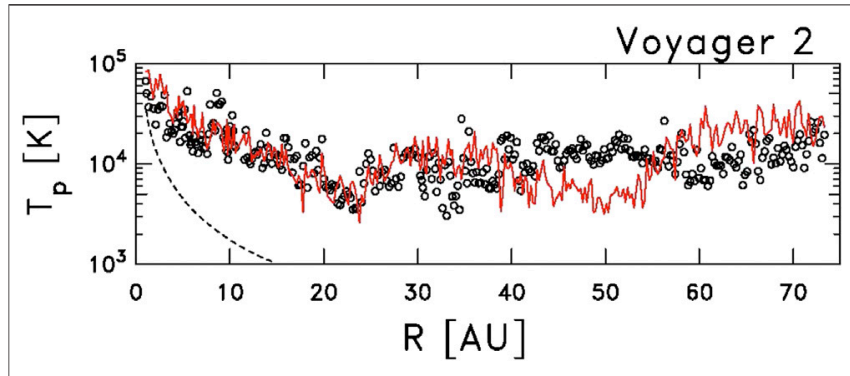

FIGURE 1 | Average solar wind proton temperature as seen by Voyager 2 (circles) and prediction of transport theory (red curve). Dashed line represents predicted proton temperature derived assuming adiabatic expansion from 1 AU (Taken from Smith et al. (2006c).

Attempts to build on this idea led to difficulties where predictions disagreed with observations. Notably, WKB theory that was used to predict the evolution of nonevolving waves with heliocentric distance was unable to account for the apparent ability of the magnetic fluctuations to remain transverse to the local mean magnetic field that rotates according to the Parker spiral (Hollweg, 1990). The local destruction and reformation of the waves would permit the newly formed waves to reorient if the dynamics were anisotropic with regard to the mean magnetic field and simulations indicate that they are (Shebalin et al., 1983; Matthaeus et al., 1990; Matthaeus et al., 1996a, Matthaeus et al., 1998; Ghosh and Goldstein, 1997; Ghosh et al., 1998a; Ghosh et al., 1998b; Cho and Vishniac, 2000; Vasquez et al., 2014). However, that means that processes normally associated with turbulence would be active and wave propagation alone could not explain the observations. At this same time, there was a growing recognition that thermal ions in the solar wind were experiencing in situ heating and the source was not recognized. It is a basic attribute of hydrodynamics (HD) that the turbulent fluctuation energy is moved to fluctuations at smaller scales where dissipation results in heating.

In this paper we review the evidence for turbulent in situ dynamics that results in the heating of the solar wind. We examine studies of solar wind fluctuations at all scales in an effort to better understand the generation, transport, and dissipation of fluctuation energy that results in the heating of the solar wind.

\section{SOLAR WIND HEATING}

It has become entirely evident that some in situ process heats the ambient thermal proton population of the solar wind. Figure 1 shows the temperature of solar wind thermal ions as measured by the Voyager 2 spacecraft (Smith et al., 2006c). The departure from adiabatic expansion from 1 AU onward clearly shows the need for some form of heating in the solar wind (Gazis et al., 1994; Richardson et al., 1995). The radial variation of the solar wind proton temperature inside $1 \mathrm{AU}$ as seen by the Helios spacecraft likewise shows the need for a heating source (Marsch et al., 1983; Schwenn, 1983; Lopez and Freeman, 1986; Arya and Freeman,
1991; Freeman et al., 1992; Totten et al., 1995; Verma et al., 1995; Vasquez et al., 2007; Hellinger et al., 2013; Lamarche et al., 2014).

Early attempts to explain the apparent heating often resembled the theories of heating that result in the acceleration of the wind from the solar corona. One notable theory, known as the cyclotron sweep mechanism, asked if it were possible to dissipate a static spectrum of magnetic fluctuations such that as the plasma moved away from the Sun and the spatial scale associated with various dissipation processes increased? Could dissipation of the observed spectrum in this manner provide the necessary energy to account for solar wind heating (Hollweg and Turner, 1978; Tu and Marsch, 1997)? It cannot (Schwartz et al., 1981). It is necessary to replace the energy that is consumed by dissipation processes or some other heating mechanism and associated source must be found to account for the observed energy budget.

Without attempting to identify the source, Vasquez et al. (2007) examined published results of the thermal proton temperature as a function of wind speed and heliocentric distance to obtain the rate of proton heating at 1 AU. They get the following expression:

$$
\varepsilon_{V \text { asquez }}=3.6 \times 10^{-5} T_{P} V_{S W}
$$

where $\epsilon_{\text {Vasquez }}$ is given in $\mathrm{J} /(\mathrm{kg} \mathrm{s}), T_{P}$ is given in Kelvin, and $V_{S W}$ is given in $\mathrm{km} \mathrm{s}^{-1}$. For typical solar wind conditions, $\epsilon_{\text {Vasquez }}$ varies from $10^{2}$ to $10^{4} \mathrm{~J} /(\mathrm{kg} \mathrm{s})$.

\section{THE TURBULENT SPECTRUM}

The overriding limitation of turbulence studies in space is that there is rarely more than one spacecraft in a region of interest. It is not possible to directly measure the nonlinear dynamics, or even the multi-dimensional spectrum, of fluctuations with a single spacecraft. There are techniques that attempt to overcome this limitation with interesting results, but in the end what is needed to make real progress in understanding any turbulent system is the ability to make measurements separated at multiple points in close proximity where the spacing of the measurements reflects the scale being studied. HelioSwarm is a proposed NASA MIDEX mission currently in a Phase-A study that will perform the multipoint measurement required to understand solar wind turbulence (Spence, 2019; Hautaluoma and Fox, 2020).

The cadence of magnetic measurements in the solar wind is generally more rapid than it is for thermal ion measurements. Therefore, there are more studies of solar wind turbulence using magnetic field measurements. The magnetic spectrum is generally divided into three ranges. There is the energycontaining range that is seen at $1 \mathrm{AU}$ at spacecraft-frame frequencies $f_{s c}<10^{-4} \mathrm{~Hz}$. These are spatial scales greater than the nominal correlation scale for the fluctuations where dynamics that are thought to originate at the Sun and persisted to the point of observation. In other words, they are direct measure of solar dynamics. The power spectrum of the energy-containing range is typically $P \sim f_{s c}^{-1}$. At lower frequencies the measured spectrum includes signals from large-scale transient solar activity, solar 


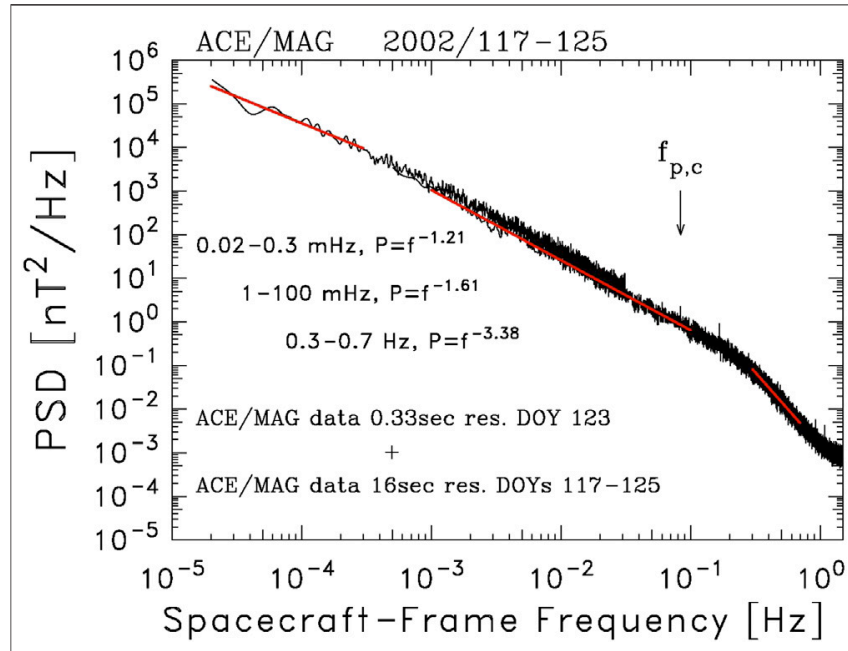

FIGURE 2 | Composite spectrum using a nine-day interval of lowresolution magnetic field data from the ACE spacecraft and a single day of high-resolution data. Frequencies $>1 \mathrm{~Hz}$ show aliasing from unresolved higher frequencies. The analysis is described in the text.

rotation, and the solar cycle. Between the correlation scale (30 min-1 h at $1 \mathrm{AU}$ ) and the dissipation scale (typically $\sim 2-5 \mathrm{~s}$ at 1 $\mathrm{AU})$ is the inertial range. This is thought to represent the nondissipative nonlinear dynamics that transport energy through the spatial scales in a conservative manner. The power spectrum of the inertial range is often characterized at $f_{s c}^{-5 / 3}$, but finite intervals of data can display a wide range of spectral indices and different theories for the dominant nonlinear dynamics predict varying spectral indices (Iroshnikov, 1964; Kraichnan, 1965; Matthaeus and Zhou, 1989; Goldreich and Sridhar, 1995; Goldreich and Sridhar, 1997; Leamon et al., 1999; Oughton and Matthaeus, 2020). At still smaller scales, the spectrum steepens and often demonstrates polarization signatures in association with the onset of dissipation.

Figure 2 shows a typical spectrum of the interplanetary magnetic field to illustrate the above description. It is a composite of two analyses using the Blackman-Tukey spectral technique (Blackman and Tukey, 1958). In the first analysis, nine days of data from days 117 through 125 of 2002 from the Advanced Composition Explorer (ACE) spacecraft with $16 \mathrm{~s}$ resolution is used to produce a measured spectrum from $2 \times$ $10^{-5}$ to $3 \times 10^{-2} \mathrm{~Hz}$. This is a series of short-lived compression and rarefaction flows, each spanning $\sim 1$ day. The frequency range from $2 \times 10^{-5}$ to $3 \times 10^{-4} \mathrm{~Hz}$ is fit with a power law to obtain $f_{s c}^{-1.21 \pm 0.02}$. This is an example of the energy-containing range. In this spectrum, there is a subtle break in the spectral index to a steeper form that occurs at $f_{s c} \sim 5 \times 10^{-4} \mathrm{~Hz}$. In the second analysis, overlayed on the same figure, $24 \mathrm{~h}$ of high resolution data from day 123 of the same mission is used to produce a measured spectrum from $5 \times 10^{-4}$ to $1.5 \mathrm{~Hz}$. This is a rarefaction interval of expanding solar wind flow. Two frequency ranges are fit to this spectrum. The first extends from $5 \times 10^{-4}$ to $0.1 \mathrm{~Hz}$ and is fit to a power law $\sim f_{s c}^{-1.61 \pm 0.01}$. This represents the inertial range. The last frequency range extends from 0.3 to $0.7 \mathrm{~Hz}$ and is fit to $f_{s c}^{-3.38 \pm 0.01}$. This represents the dissipation range. The spectral break at $\sim 0.2 \mathrm{~Hz}$ marks the onset of dissipation.

\subsection{The Inertial Range}

The inertial range is the most studied portion of the spectrum and the original source of the debate between waves or turbulence? Fluctuations are largely transverse to the local mean magnetic field, which suggests they are noncompressive (Belcher and Davis, 1971). This fluctuation anisotropy has now been shown to be equally correlated to two plasma parameters: the thermal proton $\beta_{p}=8 \pi N_{P} k_{B} T_{P} B_{0}^{-2}$ where $N_{P}$ is the proton number density, $k_{B}$ is Boltzman's constant, and $T_{P}$ is the proton temperature and the ratio of the magnetic fluctuation amplitude to the mean field strength $\delta B / B_{0}$ (Smith et al., 2006b; Pine et al., 2020b). This implies that the relative strength of the compressive fluctuations depends on one of these two parameters.

Belcher and Davis (1971) demonstrated not only the above fluctuation anisotropy, but also that the two components perpendicular to the mean field had slightly different power levels at 5:4. While this fact was widely ignored and thought to be insignificant, Bieber et al. (1996) demonstrated that the ratio of the two perpendicular components is an indication of the anisotropy of the underlying wave vectors. Using their formalism, it is easy to show that the ratio 5:4 with a nominal mean field winding angle of $45^{\circ}$ is indicative of a dominant $2 \mathrm{D}$ component meaning that the wave vectors as well as the fluctuations are largely confined to the plane perpendicular to the mean magnetic field. Subsequent analyses of many data intervals have supported the assertion that the $2 \mathrm{D}$ component is dominant in most solar wind samples (Matthaeus et al., 1990; Leamon et al., 1998a; Dasso et al., 2005; Hamilton et al., 2008; MacBride et al., 2010).

Observations combined with numerous simulations (Matthaeus et al., 1998; Cho and Vishniac, 2000; Müller and Grappin, 2005; Boldyrev et al., 2009; Beresnyak, 2011) have led to the view that the inertial range is composed largely of $2 \mathrm{D}$ turbulence with an undetermined underlying dynamic. Wave theories have resurfaced to assert that both the inertial and dissipation range is primarily composed of interacting kinetic Alfvén waves that form a turbulent plasma system (Leamon et al., 1999; Bale et al., 2005; Howes et al., 2008; Howes and Quataert, 2010; Sahraoui et al., 2010; TenBarge et al., 2013; Narita et al., 2020), and while there are problems with this interpretation (Smith et al., 2012; Roberts and Li, 2015; Vasquez et al., 2018a) it remains a popular view.

In HD, Kolmogorov (1941a) argued that the spectral transport of energy through the fluctuation spectrum was isotropic, energyconserving, and based on dynamics that are local within the spectrum. Using a structure function formalism, this lead to the famous prediction for the omnidirectional inertial-range spectrum

$$
P_{k}=C_{K} \epsilon^{2 / 3} k^{-5 / 3}
$$

where $C_{K}=1.6$ (Batchelor, 1953), $\epsilon$ is the rate of energy transport through the inertial-range spectrum, and $k$ is the wavenumber.

These ideas can be extended to magnetohydrodynamics (MHD) by employing the total energy (magnetic plus kinetic) 
and adopting a new coefficient which has been shown to apply to average solar wind conditions at 1 AU (Matthaeus and Zhou, 1989; Vasquez et al., 2007). Integrating the omnidirectional spectrum and allowing for a ratio of magnetic to kinetic energy, the extension of Eq. (2) that describes the average rate of energy transport through the spectrum at $1 \mathrm{AU}$ under changing solar wind conditions is

$$
\epsilon_{M H D}=f_{s c}^{5 / 2} P_{B}^{3 / 2} 21.8^{3} V_{S W}^{-1} N_{P}^{-3 / 2}
$$

where $P_{B}$ is the trace of the magnetic power spectral matrix in units of $\mathrm{nT}^{2} \mathrm{~Hz}^{-1}, V_{S W}$ is the solar wind speed in units of $\mathrm{km} \mathrm{s}^{-1}$, $N_{P}$ is the thermal proton density in units of $\mathrm{cm}^{-3}$, and 21.8 is a conversion factor. The factor $V_{S W}^{-1}$ converts spacecraft-frame frequency to wave number and $N_{P}^{-3 / 2}$ converts the magnetic field to units of velocity. This yields $\epsilon_{M H D}$ in units of $\mathrm{km}^{2} \mathrm{~s}^{-3}$. If we apply Eq. (3) to Figure 2 at $f_{s c}=10^{-2} \mathrm{~Hz}$, using average plasma parameters $V_{\mathrm{SW}}=400 \mathrm{~km} \mathrm{~s}^{-1}$ and $N_{p}=5 \mathrm{~cm}^{-3}$, we obtain $\epsilon_{M H D}=7.3 \times 10^{-4} \mathrm{~km}^{2} \mathrm{~s}^{-3}$ cws If we apply Eq. (1) to the same data where the average thermal proton temperature is $9.3 \times 10^{4}$ $\mathrm{K}$, we get an estimate for the average heating rate for a sample of this kind to be $\epsilon_{\text {Vasquez }}=1.3 \times 10^{-3} \mathrm{~km}^{2} \mathrm{~s}^{-3}$ which is twice the value obtained from the power spectrum and Eq. (3).

\subsection{The Dissipation Range}

In traditional HD, dissipation occurs at the smallest scales within the spectrum that are still described by fluid theory. The scale marking the spectral break and the onset of dissipation and the steepening of the spectrum depends on the rate of energy transfer through the inertial range (Smith et al., 2006a). When dissipation becomes competitive with the energy-conserving spectral transport of the inertial range, the spectrum steepens as energy is converted from fluid processes to heat.

In space, this is not the case (Leamon et al., 1999; Smith et al., 2001b; Smith et al., 2006a; Woodham et al., 2018; Pine et al., 2020a). There, the scale where dissipation sets in is determined by the ambient plasma parameters and the spectral slope associated with dissipation changes according to the rate of energy transport through the inertial range (Smith et al., 2006a; Pine et al., 2020a). Dissipation is marked by the breakdown of the fluid approximation and the necessary recovery of kinetic plasma theory. There are varying viewpoints of what dynamical processes are responsible for dissipation including cyclotron damping, Landau and transit time damping, and magnetic reconnection and those processes depend to a large degree on what form the inertial-range fluctuations take as they deliver energy to the dissipation scales (Isenberg, 1984; Isenberg, 1990; Goldstein et al., 1994; Hollweg and Isenberg, 2002; Gary et al., 2005; Isenberg and Vasquez, 2009; Parashar et al., 2009; Svidzinski et al., 2009; Markovskii et al., 2010a; Markovskii and Vasquez, 2010b; Chandran et al., 2010; Chang et al., 2011; Markovskii and Vasquez, 2011; Servidio et al., 2012; Vasquez and Markovskii, 2012; Markovskii and Vasquez, 2013a; Markovskii and Vasquez, 2013b; Bourouaine and Chandran, 2013; Chandran et al., 2013; Karimabadi et al., 2013; Kasper et al., 2013; Wu et al., 2013; Xia et al., 2013; Dalena et al., 2014; Hughes et al., 2014; Saito and Nariyuki, 2014; Servidio et al., 2014; Goldstein et al., 2015;
Isenberg and Vasquez, 2015; Servidio et al., 2015; Vásconez et al., 2015; Vasquez, 2015; Wan et al., 2015; Franci et al., 2016; Gary, Hughes and Wang, 2016; Matthaeus et al., 2016; Parashar and Matthaeus, 2016; Pucci et al., 2016; Hughes et al., 2017a; Hughes et al., 2017b; Valentini et al., 2017; Yang et al., 2017; Woodham et al., 2018). Whatever the process, dissipation in space plasmas appears to become important at scales associated with the proton inertial scale or proton cyclotron scale which are strongly correlated in the solar wind at or beyond 1 AU (Pine et al., 2020a).

At the same frequency where the spectrum steepens due to dissipation (or does not if dissipation is weak), the spectrum often becomes polarized. The polarization of the dissipation range is consistent with the removal of outward propagating Alfvén waves via cyclotron damping, but analysis shows that this is only one of several active processes (Leamon et al., 1998b; Hamilton et al., 2008). Alternatively, the polarization sense could be due to the compressive nature of 2D turbulent fluctuations (Markovskii and Vasquez, 2016).

\section{TRANSPORT THEORY}

There are three basic questions to the turbulent heating the solar wind: (1) what is the energy reservoir, (2) how does the energy get to the dissipation scales, and (3) what is the heating dynamic? Current theory holds that there are two energy reservoirs. The first is the remnant solar wind fluctuations of the energycontaining range. This includes the large-scale flow gradients of the solar wind and it is primarily responsible for the heating of the solar wind inside $10 \mathrm{AU}$. The second is the various populations of interstellar neutral atoms, primarily hydrogen, that transit the heliosphere. When ionized, they form a pickup population of suprathermal ions that excite magnetic waves at inertial-range scales close to the dissipation range. This is the dominant energy source beyond 10 AU (Zank et al., 1996; Smith et al., 2006c; Pine et al., 2020c).

It is possible to derive a transport theory describing solar wind heating without knowing the actual dissipation processes in a manner analogous to the Taylor-von Kármán approach (Taylor, 1935; von Kármán and Howarth, 1938). Under the assumption that the energy-containing range can be described to predict a rate of energy delivery to inertial-range scales, and with a description of the rate at which interstellar pickup ions excite wave energy, and assuming that all energy that is injected into the inertial range will be transported to the spectrum dissipation scales, it is possible to write equations describing the rate of solar wind heating

$$
\begin{gathered}
\frac{\mathrm{d} Z^{2}}{\mathrm{~d} r}=-\frac{A^{\prime}}{r} Z^{2}-\frac{\alpha}{U} \frac{Z^{3}}{\lambda}+\frac{\dot{E}_{P I}}{U}, \\
\frac{\mathrm{d} \lambda}{\mathrm{d} r}=-\frac{C^{\prime}}{r} \lambda+\frac{\beta}{U} Z-\frac{\beta}{U} \frac{\lambda}{Z^{2}} \dot{E}_{P I}, \\
\frac{\mathrm{d} T}{\mathrm{~d} r}=-\frac{4}{3} \frac{T}{r}+\frac{2}{3} \frac{m_{p}}{k_{B}} \frac{\alpha}{U} \frac{Z^{3}}{\lambda} .
\end{gathered}
$$

Application of these equations has focused on the energycontaining scales where $Z^{2}$ is the total fluctuation energy 
(magnetic plus kinetic) that is typically computed using hourly averages of the measured variables, $\lambda$ is the similarity scale that is typically taken to be the correlation length, and $T$ is the average temperature of the thermal protons. $A^{\prime}, C^{\prime}, \alpha$ and $\beta$, are heavily constrained by rotational symmetry, Taylor-Kármán local phenomenology, and solar wind conditions (Matthaeus et al., 1996b; Matthaeus et al., 1999). Figure 1 uses $A^{\prime}=-1.1, C^{\prime}=1.8$, $\alpha=1$, and $\beta=1$. The remaining parameters are the rate of energy injection into the turbulent spectrum by newborn interstellar PUIs $\dot{E}_{P I}$ which is obtained from other theories, the proton mass $m_{p}$, and Boltzmann's constant $k_{B}$.

The solution of these equations using parameters from $1 \mathrm{AU}$ are represented by the red curve in Figure 1. Other, sometimes more involved, versions of transport theory that follow the same general approach exist that predict a greater range of measurements and a few attempt to build specific dissipation processes into the theory (Zhou and Matthaeus, 1990a, b; Matthaeus et al., 1994; Matthaeus et al., 1996b; Matthaeus et al., 1999; Williams and Zank, 1994; Williams et al., 1995; Richardson et al., 1995; Richardson et al., 1996; Zank et al., 1996; Zank et al., 2012; Zank et al., 2017; Smith et al., 2001a; Smith et al., 2006c; Richardson and Smith, 2003; Isenberg et al., 2003; Isenberg et al., 2010; Isenberg, 2005; Breech et al., 2005; Breech et al., 2008; Breech et al., 2009; Breech et al., 2010; Oughton et al., 2006; Oughton et al., 2011; Ng et al., 2010; Usmanov et al., 2012; Usmanov et al., 2014; Usmanov et al., 2016; Usmanov et al., 2018; Adhikari et al., 2015a; Adhikari et al., b, Adhikari et al., 2017).

The intent of transport theory is that by using reasonable parameterization of the spectrum that is tightly constrained by observation, it is possible to account for the decay of the turbulent spectrum, the evolution of the break between the energy-containing and inertial range, the rate of heating, and the observed plasma temperature. While this alone may not prove that solar wind turbulence is an active heliospheric process that is responsible for the heating, it does set the bar for other theories to match.

\section{THIRD-MOMENT THEORY}

Where Kolmogorov (1941a) argued a rate of energy transport through the HD inertial range based on local dynamics and dimensional analysis, it is possible to compute a rigorous expression for the rate of energy transport in the HD inertialrange spectrum (Kolmogorov, 1941b). By assuming isotropy, homogeneity, and stationarity, the rate of energy transport through the inertial range is given by

$$
-(4 / 5) \epsilon^{H D}|\mathbf{L}|=\left\langle\left[V_{L}(\mathbf{x}+\mathbf{L})-V_{L}(\mathbf{x})\right]^{3}\right\rangle
$$

where $V_{L}$ is the component of the velocity fluctuation along the separation vector $\mathbf{L}, V_{L} \equiv \mathbf{V} \cdot \mathbf{L} / L$ where $L=|\mathbf{L}|, \epsilon^{H D}$ is the rate of energy cascade, and $\langle\ldots\rangle$ denote ensemble average. In singlespacecraft studies using the Taylor frozen-in-flx assumtion, $V_{L}$ is the radial component of the flow.

Politano and Pouquet (1998a, b) extend the Kolmogorov (1941b) analysis to include incompressible MHD turbulence using the Elsässer variables (Elsässer, 1950)

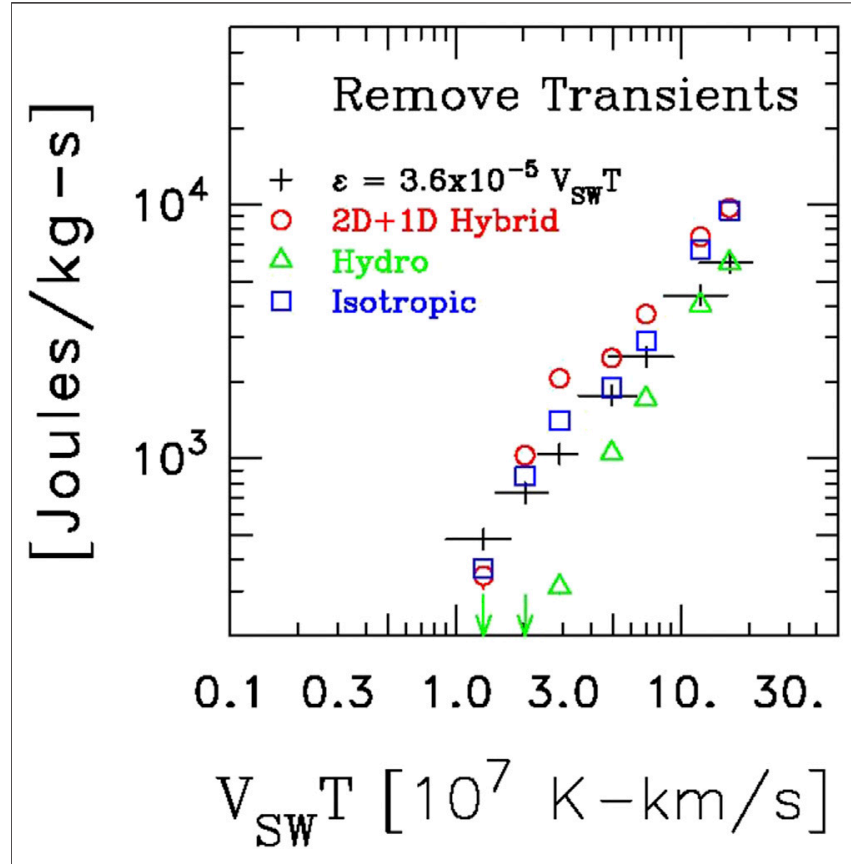

FIGURE 3 | Application of Eqs (9) and (10) years of data from the Advanced Composition Explorer (ACE) spacecraft [Reproduced from Stawarz et al. (2009)].

$$
\mathbf{Z}^{ \pm} \equiv \mathbf{V} \pm \mathbf{B} / \sqrt{4 \pi \rho}
$$

where $\rho$ is the mass density. Their expression for the spectral transport of energy in an isotropic MHD system is

$$
(4 / 3) \epsilon_{I S O}^{ \pm} V \tau=\left\langle\Delta Z_{R}^{\mp}(\tau) \sum_{i}\left[\Delta Z_{i}^{ \pm}(\tau)\right]^{2}\right\rangle
$$

where $\tau$ is the positive time lag and $\sum$ is the sum over all three vector components. The subscript " $R$ " denotes the Radial component directed from the Sun's center to the point of measurement. The total spectral transport of energy is given by

$$
\epsilon^{T}=\left(\epsilon^{+}+\epsilon^{-}\right) / 2 \text {. }
$$

It is straightforward to extend this analysis to include other geometries including $2 \mathrm{D}$. When we apply this formalism to the data represented in Figure 2, we get an average $\epsilon_{T}=3 \times$ $10^{-4} \mathrm{~km}^{2} \mathrm{~s}^{-3}$. This is half the value we obtained from Eq. (3) and a fourth the value we obtained from Eq. (1), but Stawarz et al. (2009) and Coburn et al. (2012) found better agreement between the third-moment expression and $\epsilon_{\text {vasquez }}$ when averaging a larger sample of observations.

Figure 3 compares the spectral transport of energy as computed using Eq. (9) for several different assumptions of geometry along with the scaling shown to be accurate by Vasquez et al. (2007) and the comparison is favorable. Since the results of (Vasquez et al., 2007) were obtained by examining Helios results, it represents a ground truth for the local heating rate at $1 \mathrm{AU}$. This comparison offers strong evidence that the solar wind fluctuation spectrum is not a static collection of non- 
interacting fluctuations and can be described by extensions of the HD concepts to embrace the added dynamics of MHD.

Third-moment analyses have been developed and applied to varying solar wind conditions using single- and multi-spacecraft techniques and with efforts to extend the analysis to compressive fluctuations (MacBride et al., 2005, 2008; Sorriso-Valvo et al., 2007; Marino et al., 2008; Carbone et al., 2009; Smith et al., 2009; Stawarz et al., 2009, 2010, 2011; Wan et al., 2009; Forman et al., 2010; Osman et al., 2011; Coburn et al., 2012, 2014, 2015; Banerjee et al., 2016; Hadid et al., 2017; Vasquez et al., 2018b; Smith et al., 2018; Sorriso-Valvo et al., 2018). The compressive formalisms show agreement with the incompressible formalism under most applications as would be expected since density fluctuations in the solar wind are small.

\section{INTERMITTENT HEATING}

Intermittency is generally described as the result of nonlocal dynamics contributing to the spectral transport of energy (Kolmogorov, 1962). The leading diagnostic is obtained by comparing the relative value of high-order structure functions. However, if we generalize the concept of intermittency to represent the non-steady transfer of energy, we can make a direct measure of this using the third-moment techniques described above.

Third moments computed at lags corresponding to inertialrange scales are expected to be a linear function of lag with the slope proportional to the rate of energy transport through the spectrum. Using data samples comparable in duration to the measured correlation length of the magnetic fluctuations, it is possible to show that estimates of the third-moment expressions describing spectral transfer vary significantly (Coburn et al., 2014; Coburn et al., 2015). The mean of the distribution of $\epsilon$ values agrees well with the average local heating rate of the solar wind under diverse conditions as described above, but the standard deviation is $\sim 10 \times$ the mean. Despite this variation, estimates are generally linear functions as expected and yield seemingly convergent functions. However, the correlation length for primitive variables (magnetic field, velocity, density) may not correspond to the correlation length for the third-order functions and it is this length that must be used when combining statistically independent estimates of the spectral transport rate.

Smith et al. (2018) measured the correlation length for thirdmoment expressions using the same data that produced Figure 2 and found that the correlation length was $20 \%$ of the lag value. This means that the third moment expressions decorrelate in a fraction of the scale of interest, indicating that the nonlinear dynamics of the inertial range changes significantly over any scale of interest. For instance, fluctuations seen at $0.01 \mathrm{~Hz}$ in the spacecraft frame have a spatial scale $L=400 / 0.01=4 \times 10^{4} \mathrm{~km}$ assuming a wind speed of $400 \mathrm{~km} \mathrm{~s}^{-1}$. The nonlinear dynamics associated with this scale can be expected to change significantly over $\sim L / 5=8 \times 10^{3} \mathrm{~km}$. This is significantly less than the $30 \mathrm{~min}$ to $1 \mathrm{~h}$ scale over which the primitive variables decorrelate.
The conclusion from this is that the spectral transfer rate is highly variable in both time and scale with energy being transfered to both smaller and larger scales at a mean-square rate that is $10 \times$ what is needed to account for the average heating rate. This ongoing redistribution of energy maintains the spectral form despite the fact that newborn interstellar pickup ions (PUIs) are responsible for driving the spectrum beyond $10 \mathrm{AU}$ by depositing energy at scales close to the dissipation scale (Smith et al., 2001a; Smith et al., 2006c; Pine et al., 2020c). Therefore, the nonlinear dynamics that support solar wind turbulence are much stronger than is normally inferred from the average heating rate needed to account for the observed heating.

\section{SUMMARY}

We began by discussing the multiple views that attempt to describe solar wind turbulence. To date, there is no definitive resolution to that controversy. The general morphology that describes the various ranges of the turbulent spectrum, dividing it into energy-containing, inertial, and dissipation range spans the various views, but those views offer different interpretations of the underlying dynamics. Those various dynamics each lead to predictions for the inertial-range power spectrum analogous to Eq. (2). Different forms of the transport equations, as represented here by Eqs (4)-(6), can be derived based on those same assumptions of the underlying dynamics. However, Eq. (9) embraces all underlying dynamics subject to an assumption of the underlying geometry of the wave vectors. In this way, it does provide one example of rigorous universality against which various theories of solar wind turbulence can be tested.

\section{AUTHOR CONTRIBUTIONS}

CS lead the effort in this review. BV provided material on solar wind heating and energy cascade rate estimation especially at $1 \mathrm{AU}$. Both authors contributed to the overall presentation of the review and revised the manuscript before submission.

\section{FUNDING}

CS is supported by NASA Grant NNX17AB86G and by the Advanced Composition Explorer mission. BV is supported by NASA Grant 80NSSC19K0832. CS and BV are partially supported by NASA Grant 80NSSC17K0009 and NSF SHINE AGS1622413.

\section{ACKNOWLEDGMENTS}

The data used in this analysis are available from the National Space Science Data Center and ACE Science Center. 


\section{REFERENCES}

Adhikari, L., Zank, G. P., Bruno, R., Telloni, D., Hunana, P., Dosch, A., et al. (2015b). The transport of low-frequency turbulence in the super-Alfvénic solar wind. J. Phys. Conf. Series 642, 012001. doi:10.1088/1742-6596/642/1/012001

Adhikari, L., Zank, G. P., Bruno, R., Telloni, D., Hunana, P., Dosch, A., et al. (2015a). The transport of low-frequency turbulence in astrophysical flows. Ii. Solutions for the super-alfvénic solar wind. APJ (Acta Pathol. Jpn.) 805, 63. doi:10.1088/0004-637x/805/1/63

Adhikari, L., Zank, G. P., Hunana, P., Shiota, D., Bruno, R., Hu, Q., et al. (2017). II. Transport of nearly incompressible magnetohydrodynamic turbulence from 1 to 75 au. APJ (Acta Pathol. Jpn.) 841, 85. doi:10.3847/1538-4357/aa6f5d

Arya, S., and Freeman, J. W. (1991). Estimates of solar wind velocity gradients between 0.3 and $1 \mathrm{AU}$ based on velocity probability distributions from Helios 1 at perihelion and aphelion. J. Geophys. Res. 96 (14), 183. doi:10.1029/91ja01135

Bale, S. D., Kellogg, P. J., Mozer, F. S., Horbury, T. S., and Reme, H. (2005). Measurement of the electric fluctuation spectrum of magnetohydrodynamic turbulence. Phys. Rev. Lett 94, 215002. doi:10.1103/PhysRevLett.94.215002

Banerjee, S., Hadid, L. Z., Sahraoui, F., and Galtier, S. (2016). Scaling of compressible magnetohydrodynamic turbulence in the fast solar wind. APJ (Acta Pathol. Jpn.) 829, L27. doi:10.3847/2041-8205/829/2/127

Batchelor, G. K. (1953). The theory of homogeneous turbulence. New York: Cambridge University Press.

Bavassano, B., Dobrowolny, M., Mariani, F., and Ness, N. F. (1982). Radial evolution of power spectra of interplanetary Alfvénic turbulence. J. Geophys. Res. 87, 3617. doi:10.1029/ja087ia05p03617

Belcher, J. W., and Davis, L., Jr. (1971). Large-amplitude Alfvén waves in the interplanetary medium, 2. J. Geophys. Res. 76, 3534-3563. doi:10.1029/ ja076i016p03534

Beresnyak, A. (2011). Spectral slope and Kolmogorov constant of MHD turbulence. Phys. Rev. Lett. 106, 075001. doi:10.1103/PhysRevLett.106.075001

Bieber, J. W., Wanner, W., and Matthaeus, W. H. (1996). Dominant twodimensional solar wind turbulence with implications for cosmic ray transport. J. Geophys. Res. 101, 2511-2522. doi:10.1029/95ja02588

Blackman, R. B., and Tukey, J. W. (1958). The measurement of power spectra. Mineola, NY: Dover.

Boldyrev, S., Mason, J., and Cattaneo, F. (2009). Dynamic alignment and exact scaling laws in magnetohydrodynamic turbulence. APJ (Acta Pathol. Jpn.) 699, L39. doi:10.1088/0004-637x/699/1/139

Bourouaine, S., and Chandran, B. D. G. (2013). Observational test OF stochastic heating IN LOW- $\beta$ fast-solar-wind streams. APJ (Acta Pathol. Jpn.) 774, 96. doi:10.1088/0004-637x/774/2/96

Breech, B., Cranmer, S. R., Matthaeus, W. H., Kasper, J. C., and Oughton, S. (2010). "Heating of the solar wind with electron and proton effects," in Twelfth international solar wind conference, 214-217.

Breech, B., Matthaeus, W. H., Minnie, J., Oughton, S., Parhi, S., Bieber, J. W., et al. (2005). Radial evolution of cross helicity in high-latitude solar wind. Geophys. Res. Lett. 32, L06103. doi:10.1029/2004gl022321

Breech, B., Matthaeus, W. H., Cranmer, S. R., Kasper, J. C., and Oughton, S. (2009). Electron and proton heating by solar wind turbulence. J. Geophys. Res. 114, 287. doi:10.1029/2009ja014354

Breech, B., Matthaeus, W. H., Minnie, J., Bieber, J. W., Oughton, S., Smith, C. W., et al. (2008). Turbulence transport throughout the heliosphere. J. Geophys. Res. 113, 333. doi:10.1029/2007ja012711

Carbone, V., Marino, R., Sorriso-Valvo, L., Noullez, A., and Bruno, R. (2009). Scaling laws of turbulence and heating of fast solar wind: the role of density fluctuations. Phys. Rev. Lett. 103, 061102. doi:10.1103/PhysRevLett.103. 061102

Chandran, B. D. G., Li, B., Rogers, B. N., Quataert, E., and Germaschewski, K. (2010). Perpendicular ion heating by low-frequency alfvén-wave turbulence in the solar wind. APJ (Acta Pathol. Jpn.) 720, 503. doi:10.1088/0004-637x/720/ $1 / 503$

Chandran, B. D. G., Verscharen, D., Quataert, E., Kasper, J. C., Isenberg, P. A., and Bourouaine, S. (2013). Stochastic heating, differential flow, and the alpha-toproton temperature ratio in the solar wind. APJ (Acta Pathol. Jpn.) 776, 45. doi:10.1088/0004-637x/776/1/45
Chang, O., Peter Gary, S., and Wang, J. (2011). Whistler turbulence forward cascade: three-dimensional particle-in-cell simulations. Geophys. Res. Lett. 38, 123. doi:10.1029/2011gl049827

Cho, J., and Vishniac, E. T. (2000). The anisotropy of magnetohydrodynamic alfvenic turbulence. APJ (Acta Pathol. Jpn.) 539, 273. doi:10.1086/309213

Coburn, J. T., Forman, M. A., Smith, C. W., Vasquez, B. J., and Stawarz, J. E. (2015). Third-moment descriptions of the interplanetary turbulent cascade, intermittency and back transfer. Phil. Trans. Royal Soc. A. 373, 20140150. doi:10.1098/rsta.2014.0150

Coburn, J. T., Smith, C. W., Vasquez, B. J., Forman, M. A., and Stawarz, J. E. (2014). Variable cascade dynamics and intermittency in the solar wind at $1 \mathrm{AU}$. APJ (Acta Pathol. Jpn.) 786, 52. doi:10.1088/0004-637x/786/1/52

Coburn, J. T., Smith, C. W., Vasquez, B. J., Stawarz, J. E., and Forman, M. A. (2012). The turbulent cascade and proton heating in the solar wind during solar minimum. APJ (Acta Pathol. Jpn.) 754, 93. doi:10.1088/0004-637x/754/2/93

Coleman, P. J. J., Jr. (1968). Turbulence, viscosity, and dissipation in the solar-wind plasma. APJ (Acta Pathol. Jpn.) 153, 371-388. doi:10.1086/149674

Coleman, P. J., Jr. (1966). Hydromagnetic waves in the interplanetary plasma. Phys. Rev. Lett. 17, 207-211. doi:10.1103/physrevlett.17.207

Dalena, S., Rappazzo, A. F., Dmitruk, P., Greco, A., and Matthaeus, W. H. (2014). Test-particle acceleration in a hierarchical three-dimensional turbulence model. APJ (Acta Pathol. Jpn.) 783, 143. doi:10.1088/0004-637x/783/2/143

Dasso, S., Milano, L. J., Matthaeus, W. H., and Smith, C. W. (2005). Anisotropy in fast and slow solar wind fluctuations. APJ (Acta Pathol. Jpn.) 635, L181-L184. doi:10.1086/499559

Denskat, K. U., and Burlaga, L. F. (1977). Multispacecraft observations of microscale fluctuations in the solar wind. J. Geophys. Res. 82, 2693. doi:10. 1029/ja082i019p02693

Elsässer, W. M. (1950). The hydromagnetic equations. Phys. Rev. 79, 183. doi:10. 1103/physrev.79.183

Forman, M. A., Smith, C. W., and Vasquez, B. J. (2010). Comment on "Scaling laws of turbulence and heating of fast solar wind: the role of density fluctuations". Phys. Rev. Lett. 104, 189001. doi:10.1103/PhysRevLett.104.189001

Franci, L., Landi, S., Matteini, L., Verdini, A., and Hellinger, P. (2016). Plasma beta dependence of the ion-scale spectral break of solar wind turbulence: highresolution 2D hybrid simulations. APJ (Acta Pathol. Jpn.) 833, 91. doi:10.3847/ $1538-4357 / 833 / 1 / 91$

Freeman, J. W., Totten, T., and Arya, S. (1992). A determination of the polytropic index of the free streaming solar wind using improved temperature and density radial power-law indices. EOS. Trans. AGU 73, 238.

Gary, S. P., Hughes, R. S., and Wang, J. (2016). Whistler turbulence heating of electrons and ions: three-dimensional particle-in-cell simulations. APJ (Acta Pathol. Jpn.) 816, 102. doi:10.3847/0004-637x/816/2/102

Gary, S. P., Smith, C. W., and Skoug, R. M. (2005). Signatures of Alfvén-cyclotron wave-ion scattering: Advanced Composition Explorer (ACE) solar wind observations. J. Geophys. Res 110, A07108. doi:10.1029/2004ja010569

Gazis, P. R., Barnes, A., Mihalov, J. D., and Lazarus, A. J. (1994). Solar wind velocity and temperature in the outer heliosphere. J. Geophys. Res. 99, 6561-6573. doi:10.1029/93ja03144

Ghosh, S., Matthaeus, W. H., Roberts, D. A., and Goldstein, M. L. (1998a). The evolution of slab fluctuations in the presence of pressure-balanced magnetic structures and velocity shears. J. Geophys. Res 103 (23), 691-723.704doi:10. 1029/98ja02195

Ghosh, S., Matthaeus, W. H., Roberts, D. A., and Goldstein, M. L. (1998b). Waves, structures, and the appearance of two-component turbulence in the solar wind. J. Geophys. Res. 103 (23), 705-723. doi:10.1029/98ja02194

Ghosh, S., and Goldstein, M. L. (1997). Anisotropy in Hall MHD turbulence due to a mean magnetic field. J. Plasma Phys 57, 129-154. doi:10.1017/ s0022377896005260

Goldreich, P., and Sridhar, S. (1997). Magnetohydrodynamic turbulence revisited. APJ (Acta Pathol. Jpn.) 485, 680-688. doi:10.1086/304442

Goldreich, P., and Sridhar, S. (1995). Toward a theory of interstellar turbulence. 2: strong alfvenic turbulence. APJ (Acta Pathol. Jpn.) 438, 763-775. doi:10.1086/ 175121

Goldstein, M. L., Roberts, D. A., and Fitch, C. A. (1994). Properties of the fluctuating magnetic helicity in the inertial and dissipation ranges of solar wind turbulence. J. Geophys. Res. 99 (11), 519-611. doi:10.1029/94ja00789 
Goldstein, M. L., Wicks, R. T., Perri, S., and Sahraoui, F. (2015). Kinetic scale turbulence and dissipation in the solar wind: key observational results and future outlook. Philos Trans A Math Phys Eng Sci. 373, 20140147. doi:10.1098/ rsta.2014.0147

Goldstein, M. L., Roberts, D. A., and Matthaeus, W. H. (1995). Magnetohydrodynamic turbulence in the solar wind. Annu. Rev. Astron. Astrophys 33, 283-325. doi:10.1146/annurev.aa.33.090195.001435

Hadid, L. Z., Sahraoui, F., and Galtier, S. (2017). Energy cascade rate in compressible fast and slow solar wind turbulence. APJ (Acta Pathol. Jpn.) 838, 9. doi:10.3847/1538-4357/aa603f

Hamilton, K., Smith, C. W., Vasquez, B. J., and Leamon, R. J. (2008). Anisotropies and helicities in the solar wind inertial and dissipation ranges at $1 \mathrm{AU}$. J. Geophys. Res. 113, a. doi:10.1029/2007ja012559

Hautaluoma, G., and Fox, K. (2020). NASA space weather release, 20-083. Available from: https://www.nasa.gov/press-release/nasa-selects-proposalsfor-new-space-environment-missions.

Heinemann, M., and Olbert, S. (1980). Non-WKB Alfvén waves in the solar wind. J. Geophys. Res. 85, 1311. doi:10.1029/ja085ia03p01311

Hellinger, P., Trávníček, P. M., Štverák, Š., Matteini, L., and Velli, M. (2013). Proton thermal energetics in the solar wind: Helios reloaded. J. Geophys. Res. Space Physics 118, 1351. doi:10.1002/jgra.50107

Hollweg, J. V. (1990). On WKB expansions for Alfvén waves in the solar wind. J. Geophys. Res. 95 (14), 873-814. doi:10.1029/ja095ia09p14873

Hollweg, J. V., and Isenberg, P. A. (2002). Generation of the fast solar wind: a review with emphasis on the resonant cyclotron interaction. J. Geophys. Res. 107, A071147. doi:10.1029/2001ja000270

Hollweg, J. V., and Turner, J. M. (1978). Acceleration of solar wind He++3. Effects of resonant and nonresonant interactions with transverse waves. J. Geophys. Res. 83, 97. doi:10.1029/ja083ia01p00097

Howes, G. G., Dorland, W., Cowley, S. C., Hammett, G. W., Quataert, E., Schekochihin, A. A., et al. (2008). Kinetic simulations of magnetized turbulence in astrophysical plasmas. Phys. Rev. Lett. 100, 065004. doi:10. 1103/PhysRevLett.100.065004

Howes, G. G., and Quataert, E. (2010). On the interpretation of magnetic helicity signatures in the dissipation range of solar wind turbulence. APJ (Acta Pathol. Jpn.) 709, L49. doi:10.1088/2041-8205/709/1/149

Hughes, R. S., Gary, S. P., and Wang, J. (2014). Electron and ion heating by whistler turbulence: three-dimensional particle-in-cell simulations. Geophys. Res. Lett. 41, 8681. doi:10.1002/2014gl062070

Hughes, R. S., Gary, S. P., Wang, J., and Parashar, T. N. (2017a). Kinetic Alfvén turbulence: electron and ion heating by particle-in-cell simulations. APJ (Acta Pathol. Jpn.) 847, L14. doi:10.3847/2041-8213/aa8b13

Hughes, R. S., Gary, S. P., and Wang, J. (2017b). Particle-in-cell simulations of electron and ion dissipation by whistler turbulence: variations with electron $\beta$. APJ (Acta Pathol. Jpn.) 835, L15. doi:10.3847/2041-8213/835/1/115

Iroshnikov, P. S. (1964). Turbulence of a conducting fluid in a strong magnetic field. Sov. Astron 7, 566.

Isenberg, P. A. (1990). Investigations of a turbulence-driven solar wind model. J. Geophys. Res. 95, 6437. doi:10.1029/ja095ia05p06437

Isenberg, P. A. (1984). Resonant acceleration and heating of solar wind ions: anisotropy and dispersion. J. Geophys. Res. 89, 6613. doi:10.1029/ ja089ia08p06613

Isenberg, P. A., Smith, C. W., Matthaeus, W. H., and Richardson, J. D. (2010). Turbulent heating of the distant solar wind by interstellar pickup protons in a decelerating flow. APJ (Acta Pathol. Jpn.) 719, 716-721. doi:10.1088/0004-637x/ $719 / 1 / 716$

Isenberg, P. A., Smith, C. W., and Matthaeus, W. H. (2003). Turbulent heating of the distant solar wind by interstellar pickup protons. APJ (Acta Pathol. Jpn.) 592, 564-573. doi:10.1086/375584

Isenberg, P. A. (2005). Turbulence-driven solar wind heating and energization of pickup protons in the outer heliosphere. APJ (Acta Pathol. Jpn.) 623, 502-510. doi:10.1086/428609

Isenberg, P. A., and Vasquez, B. J. (2015). Kinetic evolution of coronal hole protons by imbalanced ion-cyclotron waves: implications for measurements by Solar Probe Plus. APJ (Acta Pathol. Jpn.) 808, 119. doi:10.1088/0004-637x/808/2/119

Isenberg, P. A., and Vasquez, B. J. (2009). Preferential acceleration and perpendicular heating of minor ions in a collisionless coronal hole. APJ (Acta Pathol. Jpn.) 696, 591. doi:10.1088/0004-637x/696/1/591
Karimabadi, H., Roytershteyn, V., and Wan, M. (2013). Coherent structures, intermittent turbulence, and dissipation in high-temperature plasmas. Phys. Plasmas. 20, 012303. doi:10.1063/1.4773205

Kasper, J. C., Maruca, B. A., Stevens, M. L., and Zaslavsky, A. (2013). Sensitive test for ion-cyclotron resonant heating in the solar wind. Phys. Rev. Lett. 110, 09110. doi:10.1103/PhysRevLett.110.091102

Kolmogorov, A. N. (1941b). Dissipation of energy in locally isotropic turbulence. $R$. Soc. Lond. A 32, 16-18.

Kolmogorov, A. N. (1941a). The local structure of turbulence in incompressible viscous fluid for very large Reynolds numbers. R. Soc. Lond. A 30, 301-305.

Kolmogorov, A. N. (1962). A refinement of previous hypotheses concerning the local structure of turbulence in a viscous incompressible fluid at high Reynolds number. J. Fluid Mech 13, 82-85. doi:10.1017/s0022112062000518

Kraichnan, R. H. (1965). Inertial-range spectrum of hydromagnetic turbulence. Phys. Fluids 8, 1385-1387. doi:10.1063/1.1761412

Lamarche, L. J., Vasquez, B. J., and Smith, C. W. (2014). Proton temperature change with heliocentric distance from 0.3 to $1 \mathrm{AU}$ according to relative temperatures. J. Geophys. Res. Space Phys 119, 3267. doi:10.1002/2013ja019529

Leamon, R. J., Smith, C. W., Ness, N. F., and Wong, H. K. (1999). Dissipation range dynamics: kinetic Alfvén waves and the importance of. J. Geophys. Res 104 (22), 331-322. doi:10.1029/1999ja900158

Leamon, R. J., Matthaeus, W. H., Smith, C. W., and Wong, H. K. (1998b). Contribution of cyclotron-resonant damping to kinetic dissipation of interplanetary turbulence. Astrophys. J. Lett. 507, L181-L184. doi:10.1086/311698

Leamon, R. J., Smith, C. W., Ness, N. F., Matthaeus, W. H., and Wong, H. K. (1998a). Observational constraints on the dynamics of the interplanetary magnetic field dissipation range. J. Geophys. Res. 103, 4775-4787. doi:10. 1029/97ja03394

Lopez, R. E., and Freeman, J. W. (1986). Solar wind proton temperature velocity relationship. J. Geophys. Res.J. Geophys. Res. 92, 91.

MacBride, B. T., Forman, M. A., Smith, C. W., and ESA, S. P. (2005). "Turbulence and the third moment of fluctuations: Kolmogorov's 4/5 law and its MHD analogues in the solar wind," in Proceedings of solar wind 11: connecting Sun and heliosphere. Editors B. Fleck and T. H. Zurbuchen (The Netherlands: European Space Agency), 613-616.

MacBride, B. T., Smith, C. W., and Forman, M. A. (2008). The turbulent cascade at 1 AU: energy transfer and the third-order scaling for MHD. APJ (Acta Pathol. Jpn.) 679, 1644-1660. doi:10.1086/529575

MacBride, B. T., Smith, C. W., and Vasquez, B. J. (2010). Inertial-range anisotropies in the solar wind from 0.3 to 1 AU: Helios 1 observations. J. Geophys. Res. 115, A07105. doi:10.1029/2009ja014939

Marino, R., Sorriso-Valvo, L., Carbone, V., Noullez, A., Bruno, R., and Bavassano, B. (2008). Heating the solar wind by a magnetohydrodynamic turbulent energy cascade. APJ (Acta Pathol. Jpn.) 677, L71-L74. doi:10.1086/587957

Markovskii, S. A., and Vasquez, B. J. (2013a). "Magnetic helicity of strong 2D turbulence in the dissipation range," in Thirteenth international solar wind conference, 239.

Markovskii, S. A., and Vasquez, B. J. (2011). A short-timescale channel of dissipation of the strong solar wind turbulence. APJ (Acta Pathol. Jpn.) 739, 22. doi:10.1088/0004-637x/739/1/22

Markovskii, S. A., Vasquez, B. J., and Chandran, B. D. G. (2010a). Perpendicular proton heating due to energy cascade of fast magnetosonic waves in the solar corona. APJ (Acta Pathol. Jpn.) 709, 1003. doi:10.1088/0004-637x/709/2/1003

Markovskii, S. A., and Vasquez, B. J. (2013b). Magnetic helicity in the dissipation range of strong imbalanced turbulence. APJ (Acta Pathol. Jpn.) 768, 62. doi:10. 1088/0004-637x/768/1/62

Markovskii, S. A., and Vasquez, B. J. (2016). Magnetic helicity of ion kinetic turbulence with a nonzero electron temperature. APJ (Acta Pathol. Jpn.) 820, 15. doi:10.3847/0004-637x/820/1/15

Markovskii, S. A., and Vasquez, B. J. (2010b). The effect of spectral anisotropy of fast magnetosonic turbulence on the plasma heating at the proton kinetic scales. Phys. Plasmas 17, 112902. doi:10.1063/1.3509158

Marsch, E., Mühlhäuser, K. H., Rosenbauer, H., and Schwenn, R. (1983). On the equation of state of solar wind ions derived from Helios measurements. J. Geophys. Res. 88, 2982. doi:10.1029/ja088ia04p02982

Matthaeus, W. H., Goldstein, M. L., and Roberts, D. A. (1990). Evidence for the presence of quasi-two-dimensional nearly incompressible fluctuations in the solar wind. J. Geophys. Res. 95 (20), 673-720. doi:10.1029/ja095ia12p20673 
Matthaeus, W. H., Oughton, S., Pontius, D. H., Jr., and Zhou, Y. (1994). Evolution of energy containing turbulent eddies in the solar wind. J. Geophys. Res. 99 (19), 267-319. doi:10.1029/94ja01233

Matthaeus, W. H., Parashar, T. N., Wan, M., and Wu, P. (2016). Turbulence and proton-electron heating in kinetic plasma. Astrophys. J 7, 827. doi:10.3847/ 2041-8205/827/1/17

Matthaeus, W. H., Ghosh, S., Oughton, S., and Roberts, D. A. (1996a). Anisotropic three-dimensional MHD turbulence. J. Geophys. Res. 101, 7619. doi:10.1029/ $95 \mathrm{ja} 03830$

Matthaeus, W. H., and Goldstein, M. L. (1982). Measurement of the rugged invariants of magnetohydrodynamic turbulence in the solar wind. J. Geophys. Res. 87, 6011-6028. doi:10.1029/ja087ia08p06011

Matthaeus, W. H., Zank, G. P., and Oughton, S. (1996b). Phenomenology of hydromagnetic turbulence in a uniformly expanding medium. J. Plasma Phys 56, 659. doi:10.1017/s0022377800019516

Matthaeus, W. H., Zank, G. P., Smith, C. W., and Oughton, S. (1999). Turbulence, spatial transport, and heating of the solar wind. Phys. Rev. Lett. 82, 3444-3447. doi:10.1103/physrevlett.82.3444

Matthaeus, W. H., and Zhou, Y. (1989). Extended inertial range phenomenology of magnetohydrodynamic turbulence. Phys. Fluid. Plasma Phys 1, 1929-1931. doi: $10.1063 / 1.859110$

Matthaeus, W., Oughton, S., Ghosh, S., and Hossain, M. (1998). Scaling of anisotropy in hydromagnetic turbulence. Phys. Rev. Lett. 81, 2056. doi:10. 1103/physrevlett.81.2056

Müller, W-C., and Grappin, R. (2005). Spectral energy dynamics in magnetohydrodynamic turbulence. Phys. Rev. Lett. 95, 114502. doi:10.1103/ physrevlett.95.114502

Narita, Y., Roberts, O. W., Vörös, Z., and Hoshino, M. (2020). Transport ratios of the kinetic Alfven mode in space plasmas. Front. Phys 8, 166. doi:10.3389/fphy. 2020.00166

Ng, C. S., Bhattacharjee, A., Munsi, D., Isenberg, P. A., and Smith, C. W. (2010). Kolmogorov versus Iroshnikov-Kraichnan spectra: consequences for ion heating in the solar wind. J. Geophys. Res. 115, 124. doi:10.1029/2009ja014377

Osman, K. T., Wan, M., Matthaeus, W. H., Weygand, J. M., and Dasso, S. (2011). Anisotropic third-moment estimates of the energy cascade in solar wind turbulence using multispacecraft data. Phys. Rev. Lett. 107, 165001. doi:10. 1103/PhysRevLett.107.165001

Oughton, S., Dmitruk, P., and Matthaeus, W. H. (2006). A two-component phenomenology for homogeneous magnetohydrodynamic turbulence. Phys. Plasmas 13, 042306. doi:10.1063/1.2188088

Oughton, S., and Matthaeus, W. H. (2020). Critical balance and the physics of magnetohydrodynamic turbulence. APJ (Acta Pathol. Jpn.) 897, 37. doi:10. 3847/1538-4357/ab8f2a

Oughton, S., Matthaeus, W. H., Smith, C. W., Breech, B., and Isenberg, P. A. (2011). Transport of solar wind fluctuations: a two-component model. J. Geophys. Res. 116, 277. doi:10.1029/2010ja016365

Parashar, T. N., Shay, M. A., Cassak, P. A., and Matthaeus, W. H. (2009). Kinetic dissipation and anisotropic heating in a turbulent collisionless plasma. Phys. Plasmas 16, 032310. doi:10.1063/1.3094062

Parashar, T. N., and Matthaeus, W. H. (2016). Propinquity of current and vortex structures: effects on collisionless plasma heating. APJ (Acta Pathol. Jpn.) 832, 57. doi:10.3847/0004-637x/832/1/57

Pine, Z. B., Smith, C. W., Hollick, S. J., Argall, M. R., Vasquez, B. J., Isenberg, P. A., et al. (2020a). Solar wind turbulence from 1 to 45 AU. I. Evidence for dissipation of magnetic fluctuations using Voyager and ACE observations. APJ (Acta Pathol. Jpn.) 900, 91. doi:10.3847/1538-4357/abab10

Pine, Z. B., Smith, C. W., Hollick, S. J., Argall, M. R., Vasquez, B. J., Isenberg, P. A., et al. (2020b). Solar wind turbulence from 1 to 45 AU. III. Anisotropy of magnetic fluctuations in the inertial range using Voyager and ACE observations. APJ (Acta Pathol. Jpn.) 900, 93. doi:10.3847/1538-4357/abab11

Pine, Z. B., Smith, C. W., Hollick, S. J., Argall, M. R., Vasquez, B. J., Isenberg, P. A., et al. (2020c). Solar wind turbulence from 1 to 45 AU. IV. Turbulent transport and heating of the solar wind using Voyager observations. APJ (Acta Pathol. Jpn.) 900, 94. doi:10.3847/1538-4357/abab12

Politano, H., and Pouquet, A. (1998b). Dynamical length scales for turbulent magnetized flows. Geophys. Res. Lett. 25, 273. doi:10.1029/97gl03642

Politano, H., and Pouquet, A. (1998a). von Kármán-Howarth equation for magnetohydrodynamics and its consequences on third-order longitudinal structure and correlation functions. Phys. Rev. E 57, R21. doi:10.1103/ physreve.57.r21

Pucci, F., Vásconez, C. L., Pezzi, O., Servidio, S., Valentini, F., Matthaeus, W. H., et al. (2016). From Alfvén waves to kinetic Alfvén waves in an inhomogeneous equilibrium structure. J. Geophys. Res. Space Physics 121, 1024-1045. doi:10. 1002/2015ja022216

Richardson, J. D., and Smith, C. W. (2003). The radial temperature profile of the solar wind. Geophys. Res. Lett 30, 1206. doi:10.1029/2002gl016551

Richardson, J. D., Paularena, K. I., Lazarus, A. J., and Belcher, J. W. (1995). Evidence for a solar wind slowdown in the outer heliosphere? Geophys. Res. Lett. 22, 1469-1472. doi:10.1029/95gl01421

Richardson, J. D., Phillips, J. L., Smith, C. W., and Gray, P. C. (1996). Thermal anisotropies in the solar wind: evidence of heating by interstellar pickup ions? Geophys. Res. Lett. 23, 3259-3262. doi:10.1029/96gl02909

Roberts, O. W., and Li, X. (2015). Evidence of the ion cyclotron resonance at proton kinetic scales in the solar wind. Astrophys. J 14, 802. doi:10.1088/0004-637x/ 802/1/1

Sahraoui, F., Goldstein, M. L., Belmont, G., Canu, P., and Rezeau, L. (2010). Three dimensional anisotropickspectra of turbulence at subproton scales in the solar wind. Phys. Rev. Lett. 105, 13. doi:10.1103/physrevlett.105.131101

Saito, S., and Nariyuki, Y. (2014). Perpendicular ion acceleration in whistler turbulence. Phys. Plasmas 21, 042303. doi:10.1063/1.4870757

Schwartz, S. J., Feldman, W. C., and Gary, S. P. (1981). The source of proton anisotropy in the high-speed solar wind. J. Geophys. Res. 86, 541. doi:10.1029/ ja086ia02p00541

Schwenn, R. (1983). The average solar wind in the inner heliosphere: structures and slow variations Cp-2280. Solar Wind Five 154, 489.

Servidio, S., Valentini, F., Califano, F., and Veltri, P. (2012). Local kinetic effects in two-dimensional plasma turbulence. Phys. Rev. Lett. 108, 045001. doi:10.1103/ PhysRevLett.108.045001

Servidio, S., Valentini, F., Perrone, D., Greco, A., Califano, F., Matthaeus, W. H., et al. (2015). A kinetic model of plasma turbulence. J. Plasma Phys 81, 325810107. doi: $10.1017 /$ s0022377814000841

Servidio, S., Osman, K. T., Valentini, F., Perrone, D., Califano, F., Chapman, S., et al. (2014). Proton kinetic effects in Vlasov and solar wind turbulence. APJ (Acta Pathol. Jpn.) 781, L27. doi:10.1088/2041-8205/781/2/127

Shebalin, J. V., Matthaeus, W. H., and Montgomery, D. (1983). Anisotropy in MHD turbulence due to a mean magnetic field. J. Plasma Phys 29, 525. doi:10. $1017 /$ s0022377800000933

Smith, C. W., Mullan, D. J., Ness, N. F., Skoug, R. M., and Steinberg, J. (2001b). Day the solar wind almost disappeared: magnetic field fluctuations, wave refraction and dissipation. J. Geophys. Res 106 (18), 625-718. doi:10.1029/2001ja000022

Smith, C. W., Stawarz, J. E., Vasquez, B. J., Forman, M. A., and MacBride, B. T. (2009). Turbulent cascade at 1 AU in high cross-helicity flows. Phys. Rev. Lett. 103, 201101. doi:10.1103/PhysRevLett.103.201101

Smith, C. W., Hamilton, K., Vasquez, B. J., and Leamon, R. J. (2006a). Dependence of the dissipation range spectrum of interplanetary magnetic fluctuationson the rate of energy cascade. APJ (Acta Pathol. Jpn.) 645, L85-L88. doi:10.1086/ 506151

Smith, C. W., Isenberg, P. A., Matthaeus, W. H., and Richardson, J. D. (2006c). Turbulent heating of the solar wind by newborn interstellar pickup protons. APJ (Acta Pathol. Jpn.) 638, 508-517. doi:10.1086/498671

Smith, C. W., Matthaeus, W. H., Zank, G. P., Ness, N. F., Oughton, S., and Richardson, J. D. (2001a). Heating of the low-latitude solar wind by dissipation of turbulent magnetic fluctuations. J. Geophys. Res. 106, 8253-8272. doi:10. 1029/2000ja000366

Smith, C. W., Vasquez, B. J., Coburn, J. T., Forman, M. A., and Stawarz, J. E. (2018). Correlation scales of the turbulent cascade at 1 AU. APJ (Acta Pathol. Jpn.) 858, 21. doi:10.3847/1538-4357/aabb00

Smith, C. W., Vasquez, B. J., and Hamilton, K. (2006b). Interplanetary magnetic fluctuation anisotropy in the inertial range. J. Geophys. Res. 111, A09111. doi:10. 1029/2006ja011651

Smith, C. W., Vasquez, B. J., and Hollweg, J. V. (2012). Observational constraints on the role of cyclotron damping and kinetic Alfvén waves in the solar wind. APJ (Acta Pathol. Jpn.) 745, 8. doi:10.1088/0004-637x/745/1/8

Sorriso-Valvo, L., Carbone, F., Perri, S., Greco, A., Marino, R., and Bruno, R. (2018). On the statistical properties of turbulent energy transfer rate in the inner heliosphere. Sol. Phys 293, 10. doi:10.1007/s11207-017-1229-6 
Sorriso-Valvo, L., Marino, R., Carbone, V., Noullez, A., Lepreti, F., Veltri, P., et al. (2007). Observation of inertial energy cascade in interplanetary space plasma. Phys. Rev. Lett 99, 115001. doi:10.1103/PhysRevLett.99.115001

Spence, H. E. (2019). "HelioSwarm: unlocking the multiscale mysteries of weaklycollisional magnetized plasma turbulence and ion heating," in Fall meeting of the American Geophysical Union. 2019Abstract SH11B-04.

Stawarz, J. E., Smith, C. W., Vasquez, B. J., Forman, M. A., and MacBride, B. T. (2009). The turbulent cascade and proton heating in the solar wind at $1 \mathrm{AU}$. APJ (Acta Pathol. Jpn.) 697, 1119-1127. doi:10.1088/0004-637x/697/2/1119

Stawarz, J. E., Smith, C. W., Vasquez, B. J., Forman, M. A., and MacBride, B. T. (2010). The turbulent cascade for high cross-helicity States at $1 \mathrm{Au}$. APJ (Acta Pathol. Jpn.) 713, 920-934. doi:10.1088/0004-637x/713/2/920

Stawarz, J. E., Vasquez, B. J., Smith, C. W., Forman, M. A., and Klewicki, J. (2011). Third moments and the role of anisotropy from velocity shear in the solar wind. APJ (Acta Pathol. Jpn.) 736, 44. doi:10.1088/0004-637x/736/ $1 / 44$

Svidzinski, V. A., Li, H., Rose, H. A., Albright, B. J., and Bowers, K. J. (2009). Particle in cell simulations of fast magnetosonic wave turbulence in the ion cyclotron frequency range. Phys. Plasmas 16, 122310. doi:10.1063/1.3274559

Taylor, G. I. (1935). Statistical theory of turbulenc. Proc. R. Soc. A 151, 421-444. doi:10.1098/rspa.1935.0158

TenBarge, J. M., Howes, G. G., and Dorland, W. (2013). Collisionless damping at electron scales in solar wind turbulence. APJ (Acta Pathol. Jpn.) 774, 139. doi:10. 1088/0004-637x/774/2/139

Totten, T. L., Freeman, J. W., and Arya, S. (1995). An empirical determination of the polytropic index for the free-streaming solar wind using Helios 1 data. J. Geophys. Res 100, 13. doi:10.1029/94ja02420

Tu, C.-Y., and Marsch, E. (1997). Sol. Phys 171, 363. doi:10.1023/a:1004968327196

Usmanov, A. V., Goldstein, M. L., and Matthaeus, W. H. (2016). A four-fluid MHD model of the solar wind/interstellar medium interaction with turbulence transport and pickup protons as separate fluid. APJ (Acta Pathol. Jpn.) 820, 17. doi:10.3847/0004-637x/820/1/17

Usmanov, A. V., Goldstein, M. L., and Matthaeus, W. H. (2012). Threedimensional magnetohydrodynamic modeling of the solar wind including pickup protons and turbulence transport. APJ (Acta Pathol. Jpn.) 754, 40. doi:10.1088/0004-637x/754/1/40

Usmanov, A. V., Goldstein, M. L., and Matthaeus, W. H. (2014). Three-fluid, threedimensional magnetohydrodynamic solar wind model with eddy viscosity and turbulent resistivity. APJ (Acta Pathol. Jpn.) 788, 43. doi:10.1088/0004-637x/ $788 / 1 / 43$

Usmanov, A. V., Matthaeus, W. H., Goldstein, M. L., and Chhiber, R. (2018). The steady global corona and solar wind: a three-dimensional MHD simulation with turbulence transport and heating. APJ (Acta Pathol. Jpn.) 865, 25. doi:10.3847/ 1538-4357/aad687

Valentini, F., Vásconez, C. L., Pezzi, O., Servidio, S., Malara, F., and Pucci, F. (2017). Transition to kinetic turbulence at proton scales driven by largeamplitude kinetic Alfvén fluctuations. AઐA 599, A8. doi:10.1051/0004-6361/ 201629240

Vásconez, C. L., Pucci, F., Valentini, F., Servidio, S., Matthaeus, W. H., and Malara, F. (2015). Kinetic Alfvén wave generation by large-scale Phase mixing. APJ (Acta Pathol. Jpn.) 815, 7. doi:10.1088/0004-637x/815/1/7

Vasquez, B. J., Forman, M. A., Coburn, J. T., Smith, C. W., and Stawarz, J. E. (2018b). The turbulent cascade for high cross-helicity states at 1 au. II. minor energy. APJ (Acta Pathol. Jpn.) 867, 156. doi:10.3847/1538-4357/ aae6c6

Vasquez, B. J. (2015). Heating rate scaling of turbulence in the proton kinetic regime. APJ (Acta Pathol. Jpn.) 806, 33. doi:10.1088/0004-637x/806/1/33

Vasquez, B. J., Markovskii, S. A., and Chandran, B. D. G. (2014). Threedimensional hybrid simulation study of anisotropic turbulence in the proton kinetic regime. APJ (Acta Pathol. Jpn.) 788, 178. doi:10.1088/0004-637x/788/ $2 / 178$

Vasquez, B. J., Markovskii, S. A., and Smith, C. W. (2018a). The turbulence magnetic helicity signature in the interplanetary medium: a Blackman-Tukey and Morlet wavelet analysis. APJ (Acta Pathol. Jpn.) 855, 121. doi:10.3847/15384357/aaad0d
Vasquez, B. J., and Markovskii, S. A. (2012). Velocity power spectra from crossfield turbulence in the proton kinetic regime. APJ (Acta Pathol. Jpn.) 747, 19. doi:10.1088/0004-637x/747/1/19

Vasquez, B. J., Smith, C. W., Hamilton, K., MacBride, B. T., and Leamon, R. J. (2007). Evaluation of the turbulent energy cascade rates from the upper inertial range in the solar wind at 1 AU. J. Geophys. Res. 112, a. doi:10.1029/ 2007ja012305

Verma, M. K., Roberts, D. A., and Goldstein, M. L. (1995). Turbulent heating and temperature evolution in the solar wind. J. Geophys. Res. 100, 839. doi:10.1029/ $95 \mathrm{ja} 01216$

Völk, H. J., and Alpers, W. (1973). The propagation of Alfvén waves and their directional anisotropy in the solar wind. Astrophys. Space Sci. 20, 267.

von Kármán, T., and Howarth, L. (1938). On the statistical theory of isotropic turbulence. Proc. R. Soc. Lond. Ser. A 164, 192-215.

Wan, M., Matthaeus, W. H., Roytershteyn, V., Karimabadi, H., Parashar, T., Wu, P., et al. (2015). Intermittent dissipation and heating in 3D kinetic plasma turbulence. Phys. Rev. Lett 114, 175002. doi:10.1103/PhysRevLett.114.175002

Wan, M., Sevidio, S., Oughton, S., and Matthaeus, W. H. (2009). The third-order law for increments in magnetohydrodynamic turbulence with constant shear. Phys. Plasmas 16, 090703. doi:10.1063/1.3240333

Williams, L. L., and Zank, G. P. (1994). Effect of magnetic field geometry on the wave signature of the pickup of interstellar neutrals. J. Geophys. Res. 99 (19), 229-319. doi:10.1029/94ja01657

Williams, L. L., Zank, G. P., and Matthaeus, W. H. (1995). Dissipation of pickupinduced waves: a solar wind temperature increase in the outer heliosphere? J. Geophys. Res. 100 (17), 059-117. doi:10.1029/95ja01261

Woodham, L. D., Wicks, R. T., Verscharen, D., and Owen, C. J. (2018). The role of proton cyclotron resonance as a dissipation mechanism in solar wind turbulence: a statistical study at ion-kinetic scales. APJ (Acta Pathol. Jpn.) 856, 49. doi:10.3847/1538-4357/aab03d

Wu, P., Perri, S., Osman, K., Wan, M., Matthaeus, W. H., Shay, M. A., et al. (2013). Intermittent heating in solar wind and kinetic simulations. APJ (Acta Pathol. Jpn.) 763, L30. doi:10.1088/2041-8205/763/2/130

Xia, Q., Perez, J. C., Chandran, B. D. G., and Quataert, E. (2013). Perpendicular ion heating by reduced magnetohydrodynamic turbulence. APJ (Acta Pathol. Jpn.) 776, 90. doi:10.1088/0004-637x/776/2/90

Yang, Y., et al. (2017). Energy transfer, pressure tensor, and heating of kinetic plasma. Phys. Plasmas 24, 072306. doi:10.1063/1.4990421

Zank, G. P., Matthaeus, W. H., and Smith, C. W. (1996). Evolution of turbulent magnetic fluctuation power with heliospheric distance. J. Geophys. Res. 101 (17), 093-117. doi:10.1029/96ja01275

Zank, G. P., Adhikari, L., Hunana, P., Shiota, D., Bruno, R., and Telloni, D. (2017). Theory and transport of nearly incompressible magnetohydrodynamic turbulence. APJ (Acta Pathol. Jpn.) 835, 147. doi:10.3847/1538-4357/835/2/147

Zank, G. P., Dosch, A., Hunana, P., Florinski, V., Matthaeus, W. H., and Webb, G. M. (2012). The transport of low-frequency turbulence in astrophysical flows. I. Governing equations. APJ (Acta Pathol. Jpn.) 745, 35. doi:10.1088/0004-637x/ $745 / 1 / 35$

Zhou, Y., and Matthaeus, W. H. (1990b). Models of inertial range spectra of interplanetary magnetohydrodynamic turbulence. J. Geophys. Res. 95 (14), 881-814. doi:10.1029/ja095ia09p14881

Zhou, Y., and Matthaeus, W. H. (1990a). Transport and turbulence modeling of solar wind fluctuations. J. Geophys. Res. 95, 10291-10311. doi:10.1029/ ja095ia07p10291

Conflict of Interest: The authors declare that the research was conducted in the absence of any commercial or financial relationships that could be construed as a potential conflict of interest.

Copyright $(\odot 2021$ Smith and Vasquez. This is an open-access article distributed under the terms of the Creative Commons Attribution License (CC BY). The use, distribution or reproduction in other forums is permitted, provided the original author $(s)$ and the copyright owner(s) are credited and that the original publication in this journal is cited, in accordance with accepted academic practice. No use, distribution or reproduction is permitted which does not comply with these terms. 